\title{
"Reduced fetal urine production rate - An early marker of fetal inflammatory response syndrome in cases of preterm premature rupture of membranes: A Prospective Cohort Study"
}

\author{
Ankita Jain ${ }^{1}$, Richa Aggarwal ${ }^{2}$, Natasha Gupta ${ }^{1}$, Mohit Mehndiratta $^{1}$, Priyanka Gogoi ${ }^{1}$, \\ Prerna Batra ${ }^{1}$, and Rachna Agarwal ${ }^{1}$ \\ ${ }^{1}$ University College of Medical Sciences \\ ${ }^{2}$ Affiliation not available
}

November 2, 2020

\begin{abstract}
OBJECTIVE: To find association between fetal urine production rate (FUPR) and fetal inflammatory response syndrome (FIRS) in cases of preterm premature rupture of membranes (PPROM). DESIGN: Prospective cohort SETTING: Tertiary hospital SAMPLE: 70 pregnancies complicated by PPROM at 28-34 weeks. METHODS: Patients were managed conservatively till 34 weeks pregnancy or until spontaneous labor/chorioamnionitis. FUPR was calculated by doing serial fetal bladder volume measurements and was repeated weekly till patient delivered. During delivery, cord blood sample was taken for measuring Interleukin-6 (IL-6) levels. Placental tissue was examined for histopathologic evidence of FIRS and chorioamnionitis. Neonatal outcomes were noted as admission to NICU and severe neonatal morbidity. MAIN OUTCOME MEASURE: Measurement of FUPR antenatally and its association with development of FIRS in neonates. RESULTS: Overall prevalence of FIRS in cases of PPROM was $62.86 \%$. Mean FUPR at time of delivery was significantly reduced in neonates with evidence of FIRS as compared to Non FIRS group $(13.89 \pm 8.06 \mathrm{ml} / \mathrm{h}$ vs $25.89 \pm 4.94 \mathrm{ml} / \mathrm{h})$. Out of 41 patients with reduced FUPR prior to delivery, 39 babies had FIRS whereas only 5 out of 29 babies with normal FUPR, had FIRS ( $p$ value $<0.0001$ ). The rate of adverse neonatal morbidity was significantly high in neonates with reduced FUPR. CONCLUSION: Reduced FUPR is strongly associated with development of FIRS in cases of PPROM and hence can be used as an early predictor of adverse neonatal outcomes. FUNDING: Only institutional funds KEYWORDS: Preterm premature rupture of membrane, fetal urine production rate, fetal inflammatory response syndrome, interleukein-6 levels
\end{abstract}

\section{Title Page}

"Reduced fetal urine production rate - An early marker of fetal inflammatory response syndrome in cases of preterm premature rupture of membranes: A Prospective Cohort Study"

Ankita Jain ${ }^{1}$, Richa Aggarwal ${ }^{2}$, Natasha Gupta ${ }^{3}$, Mohit Mehndiratta ${ }^{4}$, Priyanka Gogoi ${ }^{5}$, Prerna Batra ${ }^{6}$, Rachna Agarwal ${ }^{7}$

1- Junior Resident, Department of Obstetrics \& Gynecology, University College of

Medical Sciences and GTB Hospital, University of Delhi, India

2- Associate Professor, Department of Obstetrics \& Gynecology, University College of Medical Sciences and GTB Hospital, University of Delhi, India

3- Specialist Grade 2, Department of Radiology, University College of Medical Sciences and GTB Hospital, University of Delhi, India 
4- Associate Professor, Department of Biochemistry, University College of Medical Sciences and GTB Hospital, University of Delhi, India

5-Professor, Department of Pathology, University College of Medical Sciences and GTB Hospital, University of Delhi, India

6-Professor, Department of Pediatrics, University College of Medical Sciences and GTB Hospital, University of Delhi, India

7-Professor, Department of Obstetrics \& Gynecology, University College of

Medical Sciences and GTB Hospital, University of Delhi, India

Corresponding Author - Dr Richa Aggarwal, Associate Professor, Department of Obstetrics \& Gynecology, University College of Medical Sciences and GTB Hospital, University of Delhi, Delhi 110095, India, richa.agg77@gmail.com, phone : +919891294244

Running title: Reduced FUPR- early marker of FIRS

ABSTRACT

OBJECTIVE: To find association between fetal urine production rate (FUPR) and fetal inflammatory response syndrome (FIRS) in cases of preterm premature rupture of membranes (PPROM).

DESIGN: Prospective cohort

SETTING: Tertiary hospital

SAMPLE: 70 pregnancies complicated by PPROM at 28-34 weeks.

METHODS: Patients were managed conservatively till 34 weeks pregnancy or until spontaneous labor/chorioamnionitis. FUPR was calculated by doing serial fetal bladder volume measurements and was repeated weekly till patient delivered. During delivery, cord blood sample was taken for measuring Interleukin6 (IL-6) levels. Placental tissue was examined for histopathologic evidence of FIRS and chorioamnionitis. Neonatal outcomes were noted as admission to NICU and severe neonatal morbidity.

MAIN OUTCOME MEASURE: Measurement of FUPR antenatally and its association with development of FIRS in neonates.

RESULTS: Overall prevalence of FIRS in cases of PPROM was $62.86 \%$. Mean FUPR at time of delivery was significantly reduced in neonates with evidence of FIRS as compared to Non FIRS group $(13.89 \pm 8.06 \mathrm{ml} / \mathrm{h}$ vs $25.89 \pm 4.94 \mathrm{ml} / \mathrm{h}$ ). Out of 41 patients with reduced FUPR prior to delivery, 39 babies had FIRS whereas only 5 out of 29 babies with normal FUPR, had FIRS ( $p$ value $<0.0001$ ). The rate of adverse neonatal morbidity was significantly high in neonates with reduced FUPR.

CONCLUSION: Reduced FUPR is strongly associated with development of FIRS in cases of PPROM and hence can be used as an early predictor of adverse neonatal outcomes.

FUNDING: Only institutional funds

KEYWORDS: Preterm premature rupture of membrane, fetal urine production rate, fetal inflammatory response syndrome, interleukein-6 levels

TWEETABLE ABSTRACT : A finding of reduced fetal urine production rate on in utero ultrasound examination in pregnancies complicated by preterm premature rupture of membrane is an early marker for development of fetal inflammatory response syndrome in neonates and is associated with adverse neonatal outcomes.

INTRODUCTION 
Preterm premature rupture of membranes (PPROM) is defined as spontaneous rupture of membranes before the onset of uterine contractions and before 37 weeks period of gestation. It complicates about 2-4.5\% of all deliveries but is responsible for $40 \%$ of preterm deliveries and $10 \%$ of perinatal mortality ${ }^{1,2}$. Conservative management for PPROM less than 34 weeks of gestational age is currently the accepted modality of treatment in the absence of chorioamnionitis. The rationale of conservative management in PPROM focuses mainly on prolonging the period of gestation and fetal lung maturation versus the increasing risk of potentially severe complications from maternal and neonatal infections with longer latency period. The maternal complications associated with PPROM include subclinical and clinical chorioamnionitis, premature placental separation and postpartum endometritis. Fetal complications include premature birth, infection and fetal distress due to umbilical cord compression or placental abruption.

Numerous biomarkers have been studied to detect early maternal and fetal infection, but none have been proven to be accurate in predicting the same. Early infection is not reliably predicted by investigations such as erythrocyte sedimentation rate (ESR), total leukocyte count (TLC), neutrophil count or vaginal bacterial cultures. Clinical signs like fever, fetal or maternal tachycardia appear late. Amniotic fluid culture is the most reliable test but is of limited value because of its invasive nature. Hence alternative noninvasive tests for early identification of chorioamnionitis are needed for women with PPROM.

In PPROM, micro-organisms in amniotic cavity may reach fetus and stimulate the synthesis of proinflammatory cytokines ${ }^{3}$. This leads to development of acute phase response which is almost similar to systemic inflammatory response syndrome (SIRS) seen in adults. The fetal response to intrauterine infection is termed as fetal inflammatory response syndrome (FIRS). FIRS is characterized by multi-organ involvement including the hematopoietic system, thymus, adrenal glands, heart, lungs, brain, skin, and kidneys ${ }^{4}$. It is defined by elevated IL-6 levels in cord blood or presence of characteristic histologic features of placenta which includes funisitis and chorionic vasculitis.

SIRS is characterized by redistribution of blood flow to more vital organs like heart and brain and thereby decreasing the blood supply to kidneys and hence decreased urine production. Therefore, we hypothesise that there might be a redistribution of blood flow in fetuses with FIRS with reduced blood supply to fetal kidneys, similar to that seen in SIRS. We propose to study fetal urine production rate (FUPR) measured as an early marker of fetal inflammatory response syndrome (FIRS) in patients with PPROM, so that the cases of FIRS can be picked up antenatally and timely intervention in the form of delivery can be undertaken to optimise maternal and neonatal outcomes.

\section{METHODS}

This prospective cohort study was conducted in the Department of Obstetrics and Gynaecology in University College of Medical Sciences and Guru Teg Bahadur Hospital, Dilshad Garden, New Delhi, India.

All women with a singleton pregnancy diagnosed with PPROM at gestational age $28+0$ to $33+6$ weeks between November 2018 and April 2020 were recruited. Gestational age was calculated using first day of last menstrual and confirmed by ultrasonographic dating. Women who went in spontaneous labor within 48 hours of PPROM or those with medical or obstetric pregnancy complications, or with congenital malformation in fetus were excluded from the study.

Diagnosis of PPROM was made by demonstrating vaginal pooling on sterile speculum examination and/or the $\mathrm{pH}$ evaluation of fluid collected from the posterior vaginal fornix. Following the diagnosis, expectant management was instituted as per the institutional protocol. Tablet erythromycin $(250 \mathrm{mg})$ was given orally every 6 hour for 10 days. Injection betamethasone was given 12mg, two dose, 24 hour apart for lung maturity. Patient was kept on conservative management till completed 34 weeks or if she develop features of clinical chorioamnionitis or abruptio placentae, or in case of non-reassuring fetal status. Patient was observed for signs and symptoms of chorioamnionitis. Fetal surveillance was done by daily fetal movement count, non stress test and biophysical profile. Clinical chorioamnionitis was defined as presence of fever [?] 37.8deg $\mathrm{C}$ along with two or more of following, i.e. maternal leukocytosis (TLC $>15000$ cells $/ \mathrm{mm}^{3}$ ), maternal tachycardia (heart rate $>100$ beats $/ \mathrm{min}$ ), fetal tachycardia (fetal heart rate $>160$ beats $/$ min), uterine 
tenderness and foul smelling discharge.

All patients were subjected to ultrasonographic examination within 48 hours of admission. Various parameters such as presentation, placental localization, amniotic fluid index, effective fetal weight were noted. Fetal urine production rate was calculated by doing serial fetal bladder volume measurements at an interval of 10 minutes. Bladder volume was measured on E-cube 7, Alpinion Medical Systems, BPL using a 1.0-6.0 MHz C1-6 convex array probe. Fetal bladder was first localized and focused in two planes and measurements of anteroposterior diameter (i.e. length), transverse diameter (i.e. width) and craniocaudal diameter (i.e. height) were noted, and bladder volume was generated by formula given by Campbell et al ${ }^{5}$ i.e.

Bladder volume $=\frac{4}{3} \times \pi \times$ diameter $\frac{a}{2} \times$ diameter $\frac{b}{2} \times$ diameter $\frac{c}{2}$ Diameter a: anteroposterior diameter Diameter b: transverse diameter Diameter c: craniocaudal diameter

Bladder volume was measured 3 times ultrasonographically at an interval of 10 minutes and fetal urine production rate (FUPR) was assessed by change in fetal bladder volume over time. If the second value comes out to be less than the first value as might occur with fetal voiding, then the first value was discarded and the second value was taken as initial measurement and two more values were estimated. Two values of FUPR were calculated and the mean of the resulting two values was taken. The FUPR was measured weekly till the patient delivered and the last measured FUPR was used for analysis. The FUPR was compared with the gestational age specific nomograms as given by Rabinowitz et $\mathrm{al}^{6}$. FUPR was considered reduced if the value was less than the $5^{\text {th }}$ percentile value of FUPR for that gestational age. FUPR was considered normal if it falls between $5^{\text {th }}$ and $95^{\text {th }}$ percentile values for that age.

At the time of delivery, $2 \mathrm{ml}$ cord blood sample was taken in EDTA container for measuring Interleukin-6 (IL-6) levels. The blood was centrifuged and the separated plasma was stored at -80degC till estimation of IL-6 levels (Diaclone IL-6 ELISA kit as per manufacturer's direction). The lower and upper detection limit for measurement of IL-6 levels were 1 and $200 \mathrm{pg} / \mathrm{ml}$. Some of our values of IL-6 levels were more than 200 even after putting dilution. So, we had taken those values as 200 for the calculation of mean of IL-6 levels. Placental membrane and cord tissue were collected and stored at room temperature in $10 \%$ formalin and was sent for histopathological examination to look for features suggestive of FIRS. Placental membrane was also sent for bacteriological cultures. FIRS is defined by elevated IL-6 levels in cord blood or presence of characteristic histologic features of placenta which includes funisitis and chorionic vasculitis.

Neonates were assessed by pediatrician at birth and shifted to NICU as per hospital protocol. Maternal outcomes were studied for delivery outcome, postpartum complications. Neonates were followed up and neonatal outcomes were noted as admission to NICU, duration of NICU stay and neonatal morbidity. Severe neonatal morbidity was defined as development of early onset neonatal sepsis (within 72hour), respiratory distress syndrome, bronchopulmonary dysplasia, periventricular leukomalacia, necrotizing enterocolitis or intraventricular hemorrhage.

All the procedures performed in this study on human participants were in accordance with the ethical standards of the institutional research committee (IEC-HR/2018/36/76R, dated 26/10/2018). Written Informed consent was obtained from all the individual participants included in the study. The study was funded solely by institutional funds - Intramural grant from University College of Medical Sciences, New Delhi, India.

\section{STATISTICAL ANALYSIS}

Quantitative variables were associated using Mann-Whitney Test (as data sets were not normally distributed) between the two groups. Qualitative variables were associated using Chi-Square test / Fischer's exact test. A p-value of $<0.05$ was considered statistically significant. The data analysis was done using SPSS version 21.0 .

\section{RESULTS}

During the study period, 196 women with PPROM between $28+0$ to $33+6$ weeks gestation were considered for study. Out of these, 66 had associated obstetric or medical conditions (preeclampsia, diabetes mellitus, 
intrahepatic cholestasis of pregnancy etc) and were thus excluded. Another 42 women went in spontaneous labor within 48 hours of admission and hence were not considered for study. Eight women declined to participate in the study and 10 women could not be evaluated within 48 hours of admission as shown in figure 1.

Out of 70 recruited patients with PPROM, 44 neonates were diagnosed with FIRS based on either raised IL-6 levels in cord blood or by presence of placental histologic features. Of these 44 neonates, all had raised IL-6 levels but only 16 patients had placental histologic features of FIRS. The overall prevalence of FIRS in cases of PPROM in our study was $62.86 \%$. The mean IL-6 levels in the FIRS group was $77.37+-74.51 \mathrm{pg} / \mathrm{ml}$ whereas in the Non FIRS group it was $5.75+-2.80 \mathrm{pg} / \mathrm{ml}$. Based on the presence of fetal inflammatory response syndrome, the study subjects were divided into the FIRS group and the Non FIRS group.

Both the groups were comparable with respect to maternal demographic parameters including age, education, parity, socioeconomic status. Maternal and neonatal descriptive characteristics for study participants are depicted in Table 1 which were found to be comparable between FIRS and Non FIRS group. The mean gestational age at the time of birth in the FIRS group was $32.78+-1.23$ weeks with a latency period of $8.77+-7.94$ days. These findings were comparable to the Non FIRS group where mean gestational age was $33.56+-0.89$ weeks and a latency period of $7.12+-6.19$ days. $75 \%$ of women in the FIRS group went in spontaneous labor and almost $80 \%$ women delivered vaginally. The mean birth weight was $1.59+-0.269 \mathrm{~kg}$ in the FIRS group.

The Fetal urine production rate (FUPR) at time of delivery was significantly reduced in neonates who had evidence of FIRS at birth as compared to neonates without evidence of FIRS $(13.89+-8.06 \mathrm{ml} / \mathrm{h}$ vs $25.89+-$ $4.94 \mathrm{ml} / \mathrm{h}$ ). This association of FUPR with FIRS was found to be statistically highly significant with a p-value of $<0.0001$. Out of 41 patients with reduced FUPR prior to delivery, 39 babies were subsequently found to have FIRS while out of 29 patients with normal FUPR antenatally, only 5 babies had features suggestive FIRS (Table S1).

The mean IL-6 levels in patients with reduced FUPR was $67.21+-69.86 \mathrm{pg} / \mathrm{ml}$ which was significantly higher as compared to $27.53+-60 \mathrm{pg} / \mathrm{ml}$ in patients with normal FUPR. Neonates with reduced FUPR antenatally had significantly higher rates of severe neonatal morbidity, early neonatal sepsis, prolonged NICU stay and hospital stay shown in table $2(58.54 \%, 9.05+-7.52$ days, $12.83+-8.50$ days $)$. The neonatal mortality was $4.88 \%$ in neonates with reduced FUPR. The occurrence of RDS, NEC was significantly high in neonates with recued FUPR as compared to normal FUPR.

Out of 44 neonates in the FIRS group, 27 neonates $(61.36 \%)$ had severe neonatal morbidity as compared to 8 neonates $(30.77 \%$ ) affected in the Non FIRS group. 27 babies had evidence of early onset neonatal sepsis in the FIRS group as compared to only 4 neonates in the Non FIRS group. Among the FIRS group, 15 $(34.09 \%)$ neonates had RDS, $3(6.82 \%)$ had NEC in comparison to the Non FIRS group where $4(15.38 \%)$ had RDS and 1(3.85\%) had NEC. Overall neonatal mortality was $4.55 \%$ belonging to FIRS group only (Table S2).

In our study, out of 70 patients with PPROM 42 had evidence of chorioamnionitis (clinical, bacteriological, histologic chorioamnionitis) making a prevalence of $60 \%$. Of these 42 mothers having evidence of chorioamnionitis, 38 neonates had evidence FIRS while in the absence of chorioamnionitis, only 6 out of 28 neonates had evidence of FIRS. All the 16 patients with histologic feature of FIRS had features of chorioamnionitis also on placental histopathology while none of the patients showed features of FIRS in the absence of histopathological chorioamnionitis (Figure S1, Figure S2).

Table 3 shows correlation IL-6 levels and placental histopathological features of FIRS with neonatal outcomes, it was found that out of 16 patients who had raised IL-6 levels as well as histopathological features of FIRS, $15(93.75 \%)$ neonates developed sepsis, 9 (56.25\%) had RDS, 2 (12.5\%) had NEC. In 28 cases with only raised IL-6 levels but no histologic evidence of FIRS, 12 (42.86\%) neonates had sepsis, 6 (21.43\%) developed RDS while $1(3.57 \%)$ had NEC. In 26 patients with PPROM and no biochemical and histologic evidence of 
FIRS, only 7 (26.92\%) neonates developed sepsis, 4 (15.38\%) developed RDS.

\section{DISCUSSION}

Currently there are no reliable clinical or laboratory markers to accurately predict impending neonatal infection in utero i.e. FIRS subsequent to PPROM. This study is amongst the first to show an association between FUPR and FIRS in patients of PPROM. The implications of FUPR in clinical practice are underinvestigated though it is a simple, safe and noninvasive technique of diagnosing fetal condition in utero. Using this easy to perform ultrasound examination to evaluate FUPR in cases of PPROM, we found that neonates with FIRS had lower FUPR antenatally as compared to neonates with no evidence of FIRS. In other words, reduced FUPR is significantly associated with development of FIRS in neonates born to women with PPROM.

Fetal bladder volume can be estimated ultrasonographically and the changes in bladder volume can be expressed as FUPR. In normal pregnancy, FUPR increases with gestational age i.e. from $5 \mathrm{ml} / \mathrm{hr}$ at 20 weeks of gestation to $51 \mathrm{ml} / \mathrm{hr}$ at 40 weeks $^{6}$. The ability to accurately estimate urinary flow rate non invasively in fetuses can be used for evaluation of fetal well-being. Campbell et al. first estimated human fetal urinary flow rate by calculating changes in fetal bladder volume by means of static ultrasonographic images of the fetal bladder ${ }^{5}$. The changes in bladder volume were expressed as hourly fetal urinary flow rate. Rabinowitz et al improvised this technique by taking more frequent measurements of fetal bladder volume in real time (every 2 to 5 minutes for 1 hour) and calculating the fetal urinary flow rate from the slope of the serial volume estimates by means of linear regression analysis ${ }^{6}$. Recent advances in 3D ultrasound have further improved the measurement of fetal organ volume. Few studies have been done in the past where FUPR has been used to evaluate oligohydramnios, polyhydramnios, childhood polyuric and hypercalciuric syndrome, west syndrome and as a marker of fetal well being.

We found a significant correlation between reduced FUPR and development of FIRS where it was found that out of 41 patients with reduced FUPR antenatally, 39 babies had evidence of FIRS while out of 29 patients with normal FUPR, only 5 babies had features suggestive FIRS. These findings suggest that fetuses with reduced FUPR antenatally have significantly high risk of developing FIRS which justifies our hypothesis that FUPR may be an early sign of fetal infection in cases of PPROM. Besides, we found that IL-6 levels were also markedly raised in fetuses with decreased FUPR. However very limited studies have been done in literature where FUPR has been correlated with IL- 6 levels. On searching the literature, we could find only one study which was conducted by Avitan et al in women with PPROM where they did not find any correlation between mean fetal IL-6 levels and FUPR ${ }^{7}$. However, they did not quote value of IL-6 levels in either group.

In our study the mean IL-6 levels in the FIRS group was significantly higher as compared to the Non FIRS group $(77.37+-74.51 \mathrm{pg} / \mathrm{ml}$ vs $5.75+-2.80 \mathrm{pg} / \mathrm{ml})$. All the 16 patients with histopathological evidence of FIRS had elevated IL-6 levels also. Similar findings were also found in the study by Naccasha et al, where out 15 infants whose umbilical cords had evidence of funisitis, $93 \%$ (14 of 15) had elevated IL-6 concentrations ${ }^{8}$. Another study by Mittendorf et al found that the median level of IL-6 in the newborns with funistis was 249 $\mathrm{pg} / \mathrm{ml}$ while the median IL-6 levels in the newborns without funistis was $<10 \mathrm{pg} / \mathrm{ml}^{9}$. They concluded that histological funisitis is an end-stage pathologic condition that is preceded by ever-increasing levels of IL-6 in the umbilical cord vessels.

Chorioamnionitis is the most common maternal complication after PPROM. The prevalence of chorioamnionitis in cases of PPROM in our study was $60 \%(42 / 70)$. Studies have shown that chorioamnionitis (clinical and histologic combined), complicates as many as $40-70 \%$ of preterm births with premature membrane rupture or spontaneous labor ${ }^{10}$. On comparing FUPR with chorioamnionitis, we found that reduced FUPR is associated with an increased risk of chorioamnionitis. Our findings were similar to that of Avitan et al, where they found that the mean FUPR was significantly lower in women with clinical chorioamnionitis compared to women without signs of clinical chorioamnionitis $(89.4+-0.6 \% \text { vs } 112.7+-25.2 \%)^{9}$. This was in contrast to study by Nguyen et al where the found that the presence of histologic or clinical chorioamnionitis does 
not appear to affect FUPR in women with PROM ${ }^{11}$. We further correlated FIRS with chorioamnionitis and found that FIRS is strongly associated with the presence of chorioamnionitis as majority of the newborns who had FIRS had associated evidence of chorioamnionitis in their mothers also. There might be possibility that chorioamnionitis precedes development of FIRS.

FIRS is associated with higher rates of adverse neonatal outcomes. Overall severe neonatal morbidity, risk of early onset sepsis, duration of NICU stay was significantly higher in the FIRS group as compared to the Non FIRS group. The occurrence of respiratory distress syndrome (RDS) and necrotising enterocolitis (NEC) in our study cohort was significantly higher in FIRS group (34.1\% and 6.82\%) as compared to the Non FIRS group (15.38\% and 3.85\%) suggesting FIRS as a risk factor for development of severe neonatal morbidity. Similar observations were seen by Gomez et al and Ozalkaya et al in their studies ${ }^{3,12}$. However, Zhiwei et al, Lahra et al and Watterberg et al observed significant reduction of RDS with FIRS ${ }^{13-15}$.

On correlating FUPR with neonatal outcomes, it was found that $58.54 \%$ babies with decreased FUPR antenatally had evidence of neonatal sepsis as compared to only $34.48 \%$ babies developing sepsis with normal FUPR antenatally suggesting that reduced FUPR measured antenatally is significantly associated with the risk of developing neonatal sepsis. The occurrence of RDS, NEC was significantly high in neonates with reducued FUPR as compared to normal FUPR. Only one study had been conducted so far by Avitan et al where they evaluated the association between FUPR and adverse neonatal outcome in patients with PPROM $^{7}$. They found that the lower FUPR values were associated with clinical chorioamnionitis, longer NICU stay, NEC, IVH and blood transfusion and hence can be used as early sentinel sign.

We also found that chances of developing severe neonatal morbidity were significantly high when both biochemical and histological markers of FIRS were present as compared to when only biochemical marker was elevated. However, in the absence of both, the chances of fetal morbidity was very low even in the settings of prolonged PPROM. We also correlated cord histopathology with placental histopathology and found that all 16 patients (45.71\%) with evidence of funisitis on cord histopathology had evidence of chorioamnionitis on placental histopathology suggesting that funisitis is always associated with chorioamnionitis.

Limitations of our study include small sample size of the study, however despite a small sample size, the detected association found in our study was significant. We did not measure neonatal creatinine levels and neonatal urine production in first 24 hour to correlate with reduced fetal urine production rate. Simultaneous measurement of fetal renal blood flow by renal doppler if included in our study would have enabled us to quantify renal blood flow more accurately.

Strengths of this study is its multidisciplinary approach. No study has been done to find association between fetal urine production rate and fetal inflammatory response syndrome. Both the interleukin- 6 levels and placental histopathology was used to define FIRS in our study. Very few studies in literature have used both the parameters to define FIRS in their study.

\section{CONCLUSION}

FUPR measured by simple, safe, non invasive, easy to perform ultrasonographic examination is yet underinvestigated. Reduced FUPR is an early sign of reduced fetal renal blood flow which occur in FIRS in PPROM. Further studies are required to substantiate our findings. In the interim, the establishment of pathogenesis of FIRS in causing reduced FUPR might open new avenues for early prediction of fetal inflammation leading to adverse neonatal outcome and thereby timely intervention can be taken to prevent such adverse outcomes.

Acknowledgements: We are deeply grateful to all participants in the study and to hospital staff for their cooperation.

Disclosure of Interest: The authors declare that they have no conflicts of interests.

Contribution to Authorship: AJ recruited patients, performed the study and prepared the initial draft of manuscript. RA conceptualised the study, analysed the data and helped in manuscript preparation. NG helped in measuring fetal urine production rate needed for the study. MM supervised the laboratory assay of 
Interleukein-6 levels. PG preformed the histopathological analysis of placental membranes. PB supervised neonatal assessment needed for the study. RA helped in designing the study.

Details of Ethics Approval: All the procedures performed in this study on human participants were in accordance with the ethical standards of the institutional research committee (IEC-HR/2018/36/76R, dated $26 / 10 / 2018)$. Written Informed consent was obtained from all the individual participants included in the study.

Funding: The study was funded solely by institutional funds - Intramural grant from University College of Medical Sciences, New Delhi, India.

\section{REFERENCES}

1. Aggarwal A, Pahwa S. Evaluation of the role of CRP as an early predictor of chorioamnionitis in PPROM. Int J Reprod Contracept Obstetric Gynecol. 2018;7(4):1351-56.

2. Royal College of Obstetricians and Gynaecologists. Guideline No. 44: Preterm prelabour rupture of membranes, November 2006.

3. Gomez R, Romero R, Ghezzi F, Yoon BH, Mazor M, Berry SM. The fetal inflammatory response syndrome. Am J Obstet Gynecol. 1998;179(1):194-202.

4. Gotsch F, Romero R, Kusanovic JP, et al. The fetal inflammatory response syndrome. Clin Obstet Gynecol. 2007;50(3):652-683.

5. Campbell S, Wladimiroff JW, Dewhurst CJ. The antenatal measurement of fetal urine production. J Obstet Gynaecol Br Commonw. 1973;80(8):680-6.

6. Rabinowitz R, Peters MT, Vyas S, Campbell S, Nicolaides KH. Measurement of fetal urine production in normal pregnancy by real-time ultrasonography. Am J Obstet Gynecol. 1989;161(5):1264-6.

7. Avitan T, Drukker L, Pri-Chen H, Rabinowitz R, Algur N, Schimmel MS, et al. Fetal Urine Production Rate in Preterm Premature Rupture of Membranes Is Associated with Adverse Neonatal Outcome: A Pilot Study. Gynecol Obstet Invest. 2018;83(1):57-64.

8. Naccasha N, Hinson R, Montag A, Ismail M, Bentz L, Mittendorf R. Association between funisitis and elevated interleukin-6 in cord blood. Obstet Gynecol. 2001;97(2):220-224.

9. Mittendorf R, Montag AG, MacMillan W, et al. Components of the systemic fetal inflammatory response syndrome as predictors of impaired neurologic outcomes in children. Am J Obstet Gynecol. 2003;188(6):14381446.

10. Peaceman AM, Lai Y, Rouse DJ, et al. Length of latency with preterm premature rupture of membranes before 32 weeks gestation. Am J Perinatol. 2015;32(1):57-62.

11. Nguyen TM, Meyer WJ, Gauthier DW, Myles T. Chorioamnionitis does not affect fetal urine production in patients with premature rupture of membranes. J Matern Fetal Med. 1997;6(2):96-8.

12. Ozalkaya E, Karatekin G, Topcuoğlu S, Gürsoy T, Ovalı F. Morbidity in preterm infants with fetal inflammatory response syndrome. Pediatr Int. 2016;58(9):850-4.

13. Lahra MM, Jeffery HE. A fetal response to chorioamnionitis is associated with early survival after preterm birth. Am J Obstet Gynecol. 2004;190(1):147-151.

14. Liu Z, Tang Z, Li J, Yang Y. Effects of placental inflammation on neonatal outcome in preterm infants. Pediatr Neonatol. 2014;55(1):35-40.

15. Watterberg KL, Scott SM, Naeye RL. Chorioamnionitis, cortisol, and acute lung disease in very low birth weight infants. Pediatrics 1997;99:E6.

Figure legend 
Figure 1: Study Flow Chart

Table legends

Table 1: Maternal and neonatal descriptive characteristics in FIRS and non FIRS group

Table 2: Fetal urine production rate and severe neonatal morbidity

Table 3: Association of IL-6 levels and Placental HPE with severe neonatal morbidity

TABLE 1:Maternal and neonatal descriptive characteristics in FIRS and Non FIRS group

\begin{tabular}{|c|c|c|c|}
\hline & FIRS $(\mathrm{N}=44)$ & Non FIRS $(\mathrm{N}=26)$ & p-value \\
\hline Mean age (years) & $24.73 \pm 3.180$ & $25.65 \pm 3.939$ & 0.249 \\
\hline Age distribution & Age distribution & Age distribution & Age distribution \\
\hline [?] 20 & $03(6.82 \%)$ & $02(7.69 \%)$ & \\
\hline $21-25$ & $27(61.36 \%)$ & $13(50 \%)$ & \\
\hline $26-30$ & $12(27.27 \%)$ & $08(30.77 \%)$ & \\
\hline $31-35$ & $02(4.55 \%)$ & $02(7.69 \%)$ & \\
\hline [?] 36 & 00 & $01(3.85 \%)$ & \\
\hline Education & Education & Education & Education \\
\hline Illiterate & $08(18.18 \%)$ & $02(7.69 \%)$ & 0.509 \\
\hline Primary & $02(4.55 \%)$ & $03(11.54 \%)$ & \\
\hline Middle School & $16(36.36 \%)$ & $04(15.38 \%)$ & \\
\hline High School & $11(25 \%)$ & $08(30.77 \%)$ & \\
\hline Inter & $07(15.91 \%)$ & $06(23.08 \%)$ & \\
\hline Postgraduate & 00 & $03(11.54 \%)$ & \\
\hline Socioeconomic status & Socioeconomic status & Socioeconomic status & Socioeconomic status \\
\hline Upper middle & $09(20.45 \%)$ & $13(50 \%)$ & 0.030 \\
\hline Lower middle & $32(72.73 \%)$ & $11(42.31 \%)$ & \\
\hline Upper lower & $03(6.82 \%)$ & $02(07.69 \%)$ & \\
\hline Parity & Parity & Parity & Parity \\
\hline Nullipara & $18(40.91 \%)$ & $10(38.46 \%)$ & \\
\hline Multipara & $26(59.09 \%)$ & $16(36.36 \%)$ & \\
\hline $\begin{array}{l}\text { Mean gestational at } \\
\text { birth (weeks) }\end{array}$ & $32.78 \pm 1.23$ & $33.56 \pm 0.89$ & 0.008 \\
\hline $\begin{array}{l}\text { Mean latency time } \\
\text { interval (days) }\end{array}$ & $8.77 \pm 7.94$ & $7.12 \pm 6.19$ & 0.367 \\
\hline Spontaneous labor & $33(75 \%)$ & $15(57.69 \%)$ & 0.102 \\
\hline Induction of labor & $11(25 \%)$ & $11(42.31 \%)$ & \\
\hline Mode of delivery & & & 0190 \\
\hline NVD & $35(79.55 \%)$ & $17(65.38 \%)$ & \\
\hline LSCS & $9(20.45 \%)$ & $9(34.62 \%)$ & \\
\hline Birth weight (kg) & $1.59 \pm 0.269$ & $1.85 \pm 0.309$ & 0.406 \\
\hline
\end{tabular}

TABLE 2: Fetal urine production rate and severe neonatal morbidity

\begin{tabular}{llll}
\hline & Reduced FUPR $\left(\mathbf{N}=\begin{array}{l}\text { Normal FUPR ( } \mathbf{N}= \\
\mathbf{2 9})\end{array}\right.$ & p-value \\
\hline Mean IL $-6(\mathrm{pg} / \mathrm{ml})$ & $67.21 \pm 69.86$ & $27.53 \pm 60$ & $<0.0001$ \\
Neonatal outcomes & Neonatal outcomes & Neonatal outcomes & Neonatal outcomes
\end{tabular}




\begin{tabular}{|c|c|c|c|}
\hline & $\begin{array}{l}\text { Reduced FUPR ( } N= \\
41)\end{array}$ & $\begin{array}{l}\text { Normal FUPR ( } \mathrm{N}= \\
29 \text { ) }\end{array}$ & p-value \\
\hline $\begin{array}{l}\text { Severe neonatal } \\
\text { morbidity }\end{array}$ & $24(58.54 \%)$ & $11(37.93 \%)$ & \\
\hline Sepsis & $24(58.54 \%)$ & $10(34.48 \%)$ & 0.047 \\
\hline $\begin{array}{l}\text { Duration of NICU stay } \\
\text { (days) }\end{array}$ & $9.05 \pm 7.52$ & $5.62 \pm 5.70$ & 0.103 \\
\hline $\begin{array}{l}\text { Duration of hospital } \\
\text { stay (days) }\end{array}$ & $12.83 \pm 8.50$ & $8.89 \pm 6.41$ & 0.03 \\
\hline $\begin{array}{l}\text { Respiratory distress } \\
\text { syndrome (RDS) }\end{array}$ & $13(31.70 \%)$ & $06(20.69 \%)$ & \\
\hline $\begin{array}{l}\text { Necrotizing } \\
\text { enterocolitis syndrome } \\
\text { (NEC) }\end{array}$ & $03(6.82 \%)$ & $01(3.85 \%)$ & \\
\hline Blood transfusion & $04(9.76 \%)$ & 00 & \\
\hline Mechanical ventilation & $02(4.88 \%)$ & 00 & \\
\hline Neonatal Mortality & $02(4.88 \%)$ & 00 & \\
\hline
\end{tabular}

Table 3: Association of IL-6 levels and Placental HPE with severe neonatal morbidity

\begin{tabular}{llllll}
\hline FIRS & $\begin{array}{l}\text { No. of } \\
\text { patients }\end{array}$ & $\begin{array}{l}\text { Overall } \\
\text { neonatal } \\
\text { morbidity }\end{array}$ & $\begin{array}{l}\text { Neonatal } \\
\text { Sepsis }\end{array}$ & RDS & NEC \\
\hline $\begin{array}{l}\text { IL-6 }>11 \mathrm{pg} / \mathrm{ml} \\
\mathrm{HPE} \mathrm{FIRS}+\end{array}$ & 16 & $15(93.75 \%)$ & $15(93.75 \%)$ & $09(56.25 \%)$ & $02(12.5 \%)$ \\
$\begin{array}{l}\text { IL-6 }>11 \mathrm{pg} / \mathrm{ml} \\
\text { HPE FIRS }-\end{array}$ & 28 & $12(42.86 \%)$ & $12(42.86 \%)$ & $06(21.43 \%)$ & $01(3.57 \%)$ \\
$\begin{array}{l}\text { IL-6 }<11 \mathrm{pg} / \mathrm{ml} \\
\text { HPE FIRS }+\end{array}$ & 00 & 00 & 00 & 00 & 00 \\
$\begin{array}{l}\text { IL-6 }<11 \mathrm{pg} / \mathrm{ml} \\
\text { HPE FIRS }-\end{array}$ & 26 & $08(30.77 \%)$ & $07(26.92 \%)$ & $04(15.38 \%)$ & 00 \\
\hline
\end{tabular}

\section{Hosted file}

Figure.pdf available at https://authorea.com/users/372189/articles/490311--reduced-fetalurine-production-rate-an-early-marker-of-fetal-inflammatory-response-syndrome-in-casesof-preterm-premature-rupture-of-membranes-a-prospective-cohort-study 